\title{
Accuracy Assessment of Different Registration and Imaging Methods on Image-Guided Surgery of Lateral Skull Base
}

\author{
Saleh Mohebbi ${ }^{1,2,{ }^{*}}$, Seyed Mousa Sadrehosseini ${ }^{3}$, Shabahang Mohammadi ${ }^{4}$, Thomas Lenarz ${ }^{2}$, Dirk \\ Mucha $^{5}$ and Omid Majdani ${ }^{2,6}$ \\ ${ }^{1}$ Skull Base Research Center, Iran University of Medical Sciences, Tehran, IR Iran \\ ${ }^{2}$ Otolaryngology Department of Medical Hannover School, Hannover, Germany \\ ${ }^{3}$ Brain and Spinal Cord Injury Research Center, Neuroscience Institute, Tehran University of Medical Sciences, Tehran, IR Iran \\ ${ }^{4}$ ENT Head and Neck Research Center and Department, Iran Uniersity of Medical Sciences, Tehran, IR Iran \\ ${ }^{5}$ Fiagon AG Medical Technologies, Hennigsdorf, Germany \\ ${ }^{6}$ Otolaryngology Department of Wolfsburg Clinic, Wolfsburg, Germany \\ "Corresponding author: Saleh Mohebbi, Skull Base Research Center, Iran University of Medical Science, Tehran, IR Iran. E-mail: mohebbi54@gmail.com
}

Received 2018 May 18; Accepted 2018 June 20.

\begin{abstract}
Background: The use of Image Guided Surgery (IGS) systems at lateral skull base surgery is controversial due to the limitation of the accuracy of the systems. The intraoperative accuracy of the IGS is dependent on deferent parameters, mainly the precision of the system, resolution of utilized imaging, rigidity of the reference frame/adaptor, registration method, calibration of the utilized instruments, and intraoperative movement of the reference markers.

Objectives: In this study the researchers examined the target registration error of an electromagnetic IGS system on different important structures of lateral skull base through different approaches. This study compared two registration methods and two scanning systems.

Methods: In 10 formaldehyde-fixed human cadaver heads, 69 target points were marked with titanium screws for determining target registration errors. The target points were facial nerve, lateral semi-circular canal, geniculate ganglion, superior semi-circular canal, internal auditory canal, and the foramen rotundum. Seven heads were scanned using a Cone Beam CT Scanner (CBCT) and three heads were scanned using a CT scanner. Three screws were implanted around the mastoidectomy cavity as Fiducial Markers (FM). Two different registration methods were applied, including point based registration using the FM versus surface registration. All samples were dissected via the middle cranial fossa approach, retrosigmoid approach, and transmastoid approaches.

Results: The overall accuracy of the IGS-system was $1.2 \mathrm{~mm}$ and plusmn; $0.15 \mathrm{~mm}$. Optimum accuracy was reached with CBCT-scan and the FM registration marker. Navigation using CT scans with the surface registration method, using CT scans with FM registration method, and using CBCT-scans with surface registration method had lower accuracy, respectively, yet their difference was not statistically significant $(\mathrm{P}>0.5)$. The best accuracy was noted on the facial nerve in the mastoid approach (mean $0.8 \mathrm{~mm}$ ).

Conclusions: The feasibility of the use of an electromagnetic IGS system in lateral skull base, surgery using Cone Beam CT as well as conventional CT scanner with fiducial marker registration as well as surface registration were evaluated. The accuracy was best at the lateral region of the temporal bone and decreased on further medial targets, such as lower cranial nerve and trigeminal ganglion.
\end{abstract}

Keywords: Image Guided Surgery, Navigation System, Lateral Skull Base

\section{Background}

Today, the use of IGS is widespread and accepted for many surgical procedures. In the head and neck region, the rhinologist, maxillofacial surgeons, and neurosurgeons used these devices much more routinely. The use of IGS does not diminish the surgeon's knowledge of anatomy, yet, especially in cases of altered anatomy, it helps inexperienced surgeons (1) and experienced surgeons (2) improve the outcome for extended approaches. For transnasal anterior skull base surgery, IGS systems have shown sufficient accuracy and good applicability. While at the anterior skull base an inaccuracy of approximately $2 \mathrm{~mm}$ can be tolerated, at the lateral skull base, a much higher accuracy is necessary due to the different and more complex anatomy, e.g. the boney canals of the cranial nerves, vital arteries, and veins. In the temporal bone, the position of the hidden labyrinthine organ and facial nerve has been challenging for the IGS-technology. The structures are very small in size and almost no safety region is tolerable while drilling the surrounding bone for ap- 
proaching the lateral skull base. Therefore, IGS systems utilized in this field need to provide high accuracy. One demanding component of the accuracy of the IGS is the registration process. Commonly, all systems need to register the image data to patients' real coordinates at the beginning of the surgery. Two different and commonly used registration strategies are surface marking and Fiducial Marking (FM) (3). Surface registration is applicable for rhinology and anterior skull base surgery and is widely used, because of acceptable accuracy for this type of surgery and easy applicability in any patient (3). By this method, the system registers a different number of points on the patient's midface before starting the intervention. However, in the temporal bone and lateral skull base, surface registration approaches do not show good accuracy. In these cases, fiducial marker-based registration is the gold standard (4). For this purpose, pre-imaging implantation of the fiducial markers is mandatory. In lateral skull base cases, the FM can be implanted around the mastoid cavity. After implantation imaging, CBCT or CT can be acquired and these images are used during surgery for registration and target finding. If intraoperative imaging is available, the entire setup can occur during a single session; otherwise, a two-step surgery is necessary. However, the implantation of three to four fiducial markers, usually $1.5 \times 6 \mathrm{~mm}$ or similar size titanium screws, can occur in local anesthesia. Surface registration has also been tried on lateral skull base navigation (5).

There are two main tracking technologies available: optoelectronics and electromagnetic. Each has some advantages and disadvantages. Optoelectronic IGS systems are reliable and accurate yet need a constant line of site between the camera and surgical field. Electromagnetic systems do not need a direct line of sight, yet, tracking might get degraded by metallic objects in the surgical field (6).

During the last decade, several companies have worked to find the best methods and systems for performing image guidance on the complex anatomy of the lateral skull base. However, safe use of the image-guided surgery in this area is still challenging.

\section{Objectives}

In this study, the authors evaluated the accuracy of electromagnetic tracking system of the Fiagon Navigation system (Fiagon GmbH, Hennigsdorf, Germany) when using different imaging methods and registration methods on the lateral skull base of the cadaveric specimen.

\section{Methods}

The Fiagon-IGS system, essentially consists of a hardware module, application software, magnetic field gener- ator, electromagnetic sensors that is fixed on the head and a pointer. The flexible pointer includes the sensor coils at the tip of the instrument and, therefore, the shape of the metallic instrument can be adapted to the needs of the surgeon by curving the shaft of the instrument without losing accuracy (Figure 1).

Imaging was performed with the intraoperative Cone Beam CT (Xoran XCAT ENT, Annarbor, MI, USA) and the CT $\operatorname{scan}$ (GE Lightspeed 16).

Ten formaldehyde-fixed anatomical human cadavers were provided by the Department of Anatomy, Hannover Medical School. According to the EU guideline on "Good Clinical Practice", the Declaration of Helsinki of the World Medical Association, and the Occupational Regulation for German Physicians (MBO-A 1997), vote of the ethics committee was not necessary. All samples were prepared with a standard surgical technique for Middle Cranial Fossa (MCF), Retrosigmoid (RS) and Mastoidectomy preparation for Translabyrinthine approach (TL). Different target points were marked with screws (titanium screws with a diameter of $2 \mathrm{~mm}$ ) for all approaches. The superior semicircular canals (sup-SCC) and Geniculate Ganglion (GG) were approached through the MCF. The Internal Auditory Canal (IAC), Lower Cranial Nerves (LCN), and Trigeminal Ganglion (V-CN) were marked through the retrosigmoid approach. Through mastoidectomy cavity, the lateral Semicircular Canal (Lat-SCC) and facial nerve (VII-CN) were found and marked. Three points were marked around the mastoidectomy margin for FM (anterosuperior, posterosuperior, and posteroinferior side) and were marked and supplied with screws. Due to the use of the specimens for education at the anatomy department, some target points were not accessible or presumable. At least six to nine target sites were marked in all phantoms. Seven samples were scanned by Cone Beam CT; the same was used for intraoperative scanning and three samples were scanned with the conventional CT scanner.

The data was stored on a CD-ROM. The data sets of the CBCT had the following specifications:

- Axial slices

- Resolution in each layer: $0.3 \mathrm{~mm} \times 0.3 \mathrm{~mm}(812 \times 812$ pixels)

- Layer distance: $0.3 \mathrm{~mm}$

The data sets of the CT had the following specifications:

- Axial slices

- Resolution in each layer: $0.4 \mathrm{~mm} \times 0.4 \mathrm{~mm}(512 \times 512$ pixels)

- Layer distance: $0.5 \mathrm{~mm}$ 


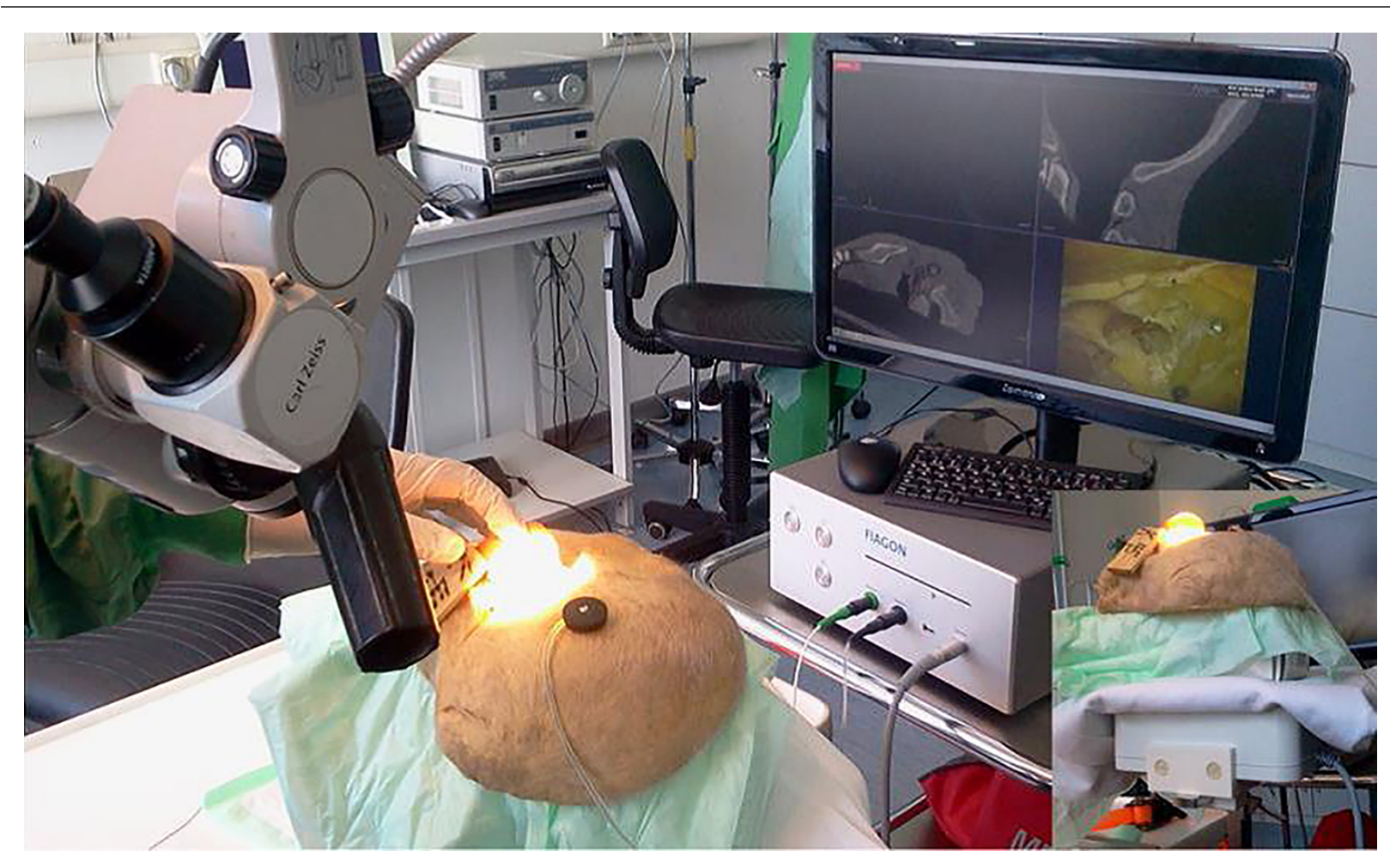

Figure 1. System setup, a hardware module, application software, the magnetic field, generator and specific sensors that fix the head

The navigation data set was imported to the IGS system and the clearly visible titanium screws, which served partly as registration and partly as target points for evaluation, were marked manually in the image data. Hereby, the image data coordinate system was determined and these positions were saved as reference points $r(i)$, where $(i)$ is the index of the markers.

During the course of the evaluation, the phantom was equipped with a localizer that was fixed to the skull with one bone screw. It was placed on the navigation head rest. The complete setup was similar to the intraoperative real patient treatment.

The software used in the study was Fiagon Navigation release 3.7.4 measure. In all functions, the software is equal to clinical release 3.7.4. It additionally allows for recording the position and orientation values of the instrument during each measure cycle to a log-file (text-file). The recorded values were the position and orientation of the instrument in the coordinate system of the registered image data set; thereby, these positions could be directly compared to the reference points.

The registration process was carried out according to instructions for use, as there is:

- Marking 3 marker in the image data set
- Touching the corresponding points on the phantom with the pointer instrument (Fine Pointer)

Thereafter, each titanium marker was touched with the Fine Pointer and held still (freehand) on the marker. The position of the pointer was recorded over 100 measuring cycles with each marker. From this data, the following was computed:

1. Mean position $\mathrm{p}(\mathrm{i})$ at each marker $\mathrm{i}$ as the mean out of the 100 recorded position values, resp.

2. Deviation e (i) at each marker $i$ as the length of the vector between measured position $p(i)$ and reference point $\mathrm{r}(\mathrm{i})$

3. Mean and standard deviation over all calculated deviations e (i) computed in step 2.

Furthermore, a screenshot of the pointer on the screw was taken with the screenshot function of the system. The following items were used for evaluation and data handling, which are shown in Table 1. 


\section{Results}

The current research evaluated the target accuracy error of the device on different target points by a fine-tip probe on the lateral skull base (Figure 2). The researchers examined two different imaging modalities and two different registration methods.

The best combination of methods in this study was using CBCT imaging and FM-based registration with an average error of $1.05 \mathrm{~mm} \pm 0.49$. The accuracy of CT scan with FM registration was $1.24 \pm 0.48 \mathrm{~mm}$ without a significant difference $(\mathrm{P}=0.350913)$ between the two methods.

The accuracy was checked also with surface registration on the lateral part of the skull (the mastoid and temporal area). The findings of this registration type on the CT scanning have shown better results than the CBCT scanning system. The mean accuracy was $1.21 \pm 0.50 \mathrm{~mm}$ on the CT and $1.37 \pm 0.59$ on the CBCT image. The differences were statistically non-significant $(\mathrm{P}=0.46423118)$. The accuracy of the system in different imaging systems and different registration methods are shown in Table 2.

\begin{tabular}{lcc}
\hline \multicolumn{2}{l}{ Table 1. The Items Were Used for the Evaluation and Data Handling } \\
\hline No. & Items & Specification / SN \\
\hline $\mathbf{1}$ & Navigation unit & 11 - 0012 \\
$\mathbf{2}$ & Navigation head rest & FG 0012 \\
\hline $\mathbf{3}$ & Navigation software & R 11- \\
$\mathbf{4}$ & Localizer bone screw & O 12- \\
$\mathbf{5}$ & Fine pointer & Microsoft excel 2013 \\
\hline $\mathbf{6}$ & Computation &
\end{tabular}

Regarding the difference between the two registration methods, FM-based registration was better than surface registration on $\mathrm{CBCT}$; however, the difference of the significance was 0.813 and not significant. In the CT scan method, the registration method did not show any significant difference $(P=0.198)$, even though the FM-base registration accuracy was better.

The difference of CBCT and CT scan imaging system and two types of registration methods (FM and surface) were checked. However, the CBCT with FM registration was better, yet no significant difference was seen between them (Figure 3).

In the CBCT scanning on the basis of FM registration method, the average was $1.05 \pm 0.49 \mathrm{~mm}$. The best point of accuracy was on the facial nerve $(0.8 \mathrm{~mm} \pm 0.43 \mathrm{~mm})$. The accuracy of the IGS system with this method on the other point was as follows: at Geniculate Ganglion (GG) 1.15 $\mathrm{mm} \pm 0.55 \mathrm{~mm}$, Superior Semicircular Canal (Sup_SCC) 1.2 $\mathrm{mm} \pm 0.45$, Lateral Semicircular Canal (Lat-SCC) $1.1 \mathrm{~mm} \pm$ 0.53 , Internal Auditory Canal (IAC) $1.3 \mathrm{~mm} \pm 0.5$, and Lower
Cranial Nerve (LCN) $1.5 \mathrm{~mm} \pm 0.49$ (Table 2). The least accurate point was detected on the trigeminal ganglion site (1.54 $\mathrm{mm} \pm 0.1 \mathrm{~mm})$ (Figure 4).

Changing the imaging system to regular CT scan with FM registration decreased the accuracy to $1.24 \pm 0.48 \mathrm{~mm}$. The most accurate point was GG $(1.0 \mathrm{~mm} \pm 0.44 \mathrm{~mm})$, the other points were facial nerve $(1.3 \mathrm{~mm} \pm 0.44 \mathrm{~mm})$, SupSCC (1.5 mm $\pm 0.7 \mathrm{~mm})$, Lat-SCC (1.3 mm \pm 0.44$)$, IAC (1.0 $\mathrm{mm} \pm 0.2 \mathrm{~mm}$ ), lower cranial nerve (LCN) $1.8 \mathrm{~mm} \pm 0.1 \mathrm{~mm}$ (Table 2).

In the $\mathrm{CBCT}$ imaging with surface registration, the result showed the accuracy on the GG $(1.7 \mathrm{~mm} \pm 0.6 \mathrm{~mm})$, facial nerve $(1.0 \mathrm{~mm} \pm 0.5 \mathrm{~mm})$, Sup-SCC $(1.4 \mathrm{~mm} \pm 0.6 \mathrm{~mm})$, Lat-SCC (1.2 mm $\pm 0.9 \mathrm{~mm}), \operatorname{IAC}(1.2 \mathrm{~mm} \pm 0.33 \mathrm{~mm})$, and LCN $(1.4 \mathrm{~mm} \pm 1 \mathrm{~mm})$. The accuracy on target markers of CT by surface registration were on $\mathrm{GG}(1.2 \mathrm{~mm} \pm 0.5)$, facial nerve $(1.0 \mathrm{~mm} \pm 0.4 \mathrm{~mm})$, Sup-SCC $(1.2 \mathrm{~mm} \pm 0.5 \mathrm{~mm})$, LatSCC $(0.9 \mathrm{~mm} \pm 0.05 \mathrm{~mm}), \operatorname{IAC}(1.6 \mathrm{~mm} \pm 0.34 \mathrm{~mm})$, and LCN (1.0 mm $\pm 0.6 \mathrm{~mm}$ ), see Table 2 .

The overall accuracy of the system for all registration and imaging methods was $1.2 \mathrm{~mm} \pm 0.15$ (Figure 5). The accuracy of the systems was checked also on more medial anatomical areas, such as the trigeminal ganglion in three samples. The accuracy was less than the other areas with all methods ( $1.6 \mathrm{~mm} \pm 0.1 \mathrm{~mm}$ ). Placing the navigation probe on the screw in deep anatomical areas through the limited approach size was difficult; this fact needs to be considered as it was not included in the assessment.

There were some problems in registration in the surface area because of the metallic distracter instrument. However, the researchers did not notice any magnetic field disturbance in the surgical field as mastoid, MCF or retrosigmoid.

\section{Discussion}

Using the intraoperative IGS-system has advantages in different fields, such as sinus and anterior skull-based surgery (7). For lateral skull-based surgery, a more accurate system is necessary because of the anatomical complexity in the temporal bone. An accuracy of less than $1 \mathrm{~mm}$ is reported to be acceptable and an accuracy of $0.5 \mathrm{~mm}$ or less is desirable (8).

For the clinical validation of IGS systems, accurate identification of the anatomic points as targets was performed repeatedly. Cadaveric studies have been performed to credit the system for clinical studies (9). The lowest limit of error is necessary to increase the safety and effectiveness for use.

Different navigation systems, such as magnetic or optical systems, have their advantages and disadvantages. In the electromagnetic Fiagon system, problems were not encountered with disturbing the camera-field view, as with 


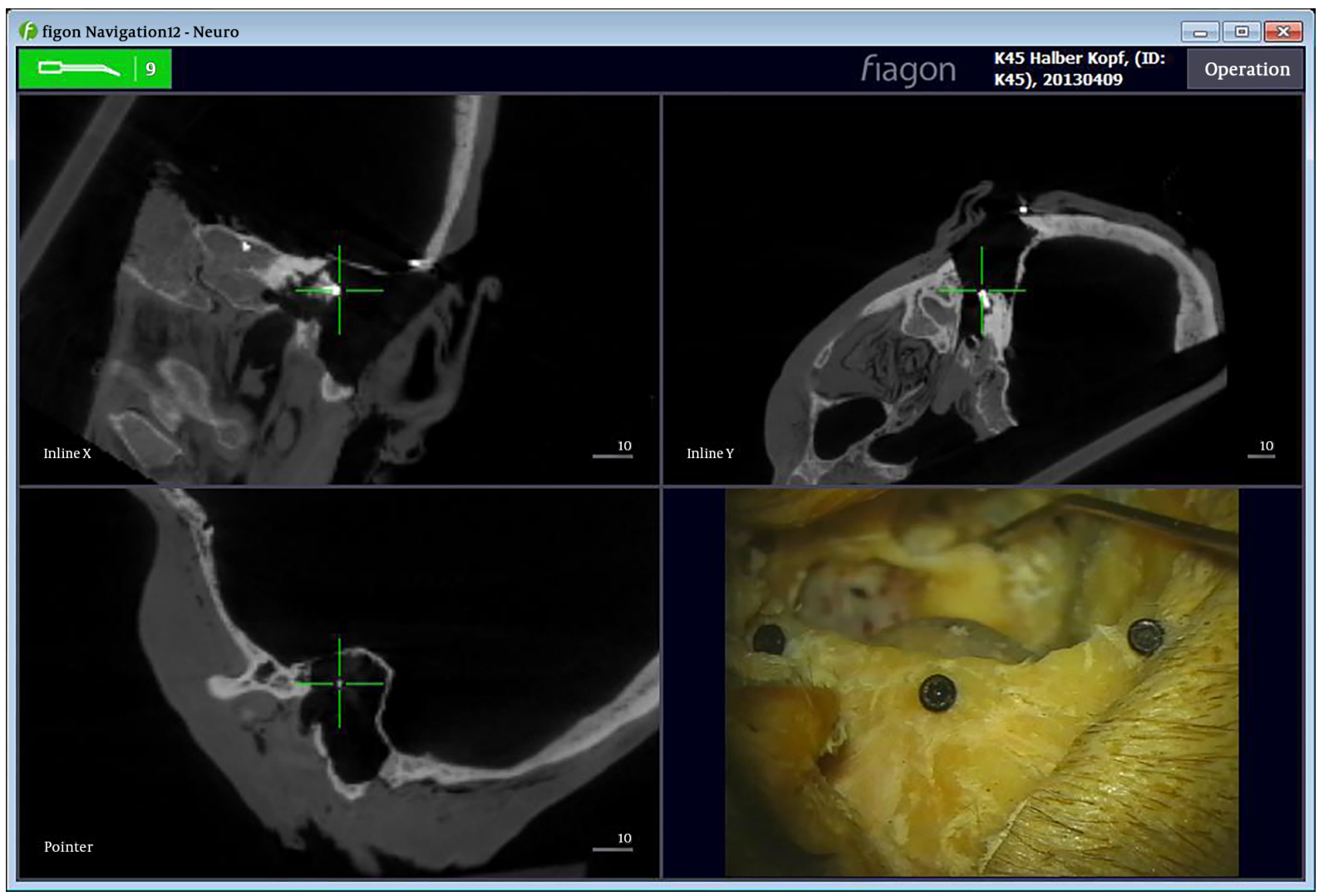

Figure 2. A screen shot of target point looking by fine probe tip

\begin{tabular}{lcccc}
\hline Table 2. Mean Accuracy of Different Imaging Techniques and Registration Methods & & & \\
\hline Imaging and Registration & Target Markers & Average & SD & 0.49 \\
\hline CBCT by FM & 13 registration & 1.05 & 1.21 & 0.50 \\
CT by Surface & 3 registration & 3 sample & 1.24 & 0.48 \\
CT by FM & 3 registration & 3 sample & 1.37 & 0.59 \\
CBCT by Surface & 8 registration & 5 sample \\
\hline
\end{tabular}

Abbreviations: CBCT, cone beam CT scanner; FM, fiducial markers; SD, standard devition.

optical tracking systems, yet there were problems with superficial registration. During surgery, all target points were far from the surface, so the system is applicable. The microscope distance was preserved near $300 \mathrm{~mm}$ to surgical field, as previously done by Bernardeschi et al. (10).

The bone implanted markers, Fiducial marker, are accepted as the gold standard for registration $(9,11)$. The totally fixed titanium screw with central head cavity was very suitable for accurate registration and point defining and decreased the error of measurement. The screws were clearly visible in the CBCTand CT scan images. As in case of all implanted markers, this is an invasive procedure, and imaging for IGS-use needs to be acquired after implantation. However, in case of surface registration, there is no need for the invasive procedure and repeating the imaging. In this study, the researchers compared both registration methods. Surface registration is popular in rhinology, yet for lateral skull base, more research is still required (5, 12).

It has been noted that for enhanced registration, at least six markers are necessary (4), yet with new evolution in hardware and software, three markers were successfully used.

Researchers usually use different target points to ad- 


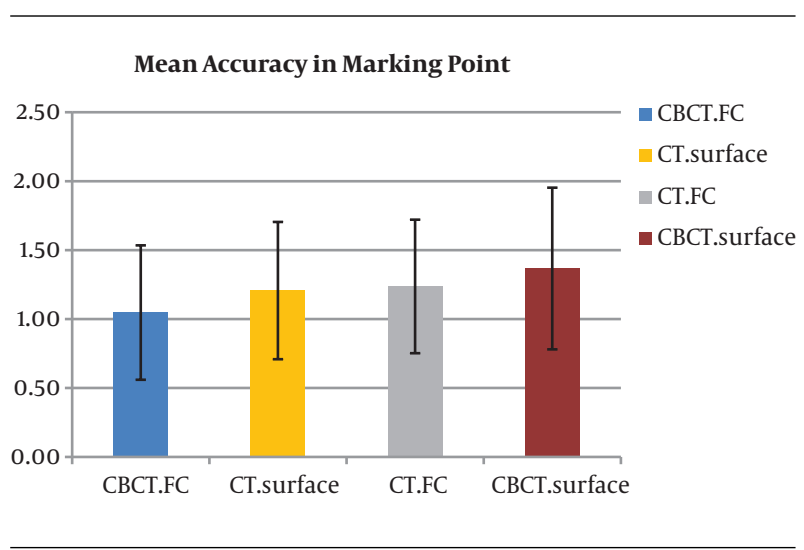

Figure 3. Mean accuracy of different imaging and registration protocols

dress the target registration error. The BAHA abutment (9), middle ear and cranial base structures $(10,13)$ and different points of interest, selected in popular lateral skull base approaches, have been examined to define the target registration error in the depth of the sites. This study selected the Sup.SCC and GG through the MCF approach, the facial nerve and lateral semicircular (Lat.SCC) through the transmastoidal approach, and the IAC and lower cranial nerves and trigeminal ganglion position in the retrosigmoid approach. Finding these landmarks during surgery is vital and can help surgeons perform the surgery safely and with lower morbidity. The current researchers used a specific titanium screw that fitted the probe tip in these eight target areas.

A lower stat of error was noted in FM marker registration by CBCT scanning, followed by surface marking registration, FM registration by CT scan and surface marking registration by CBCT, respectively (Table 1). There are no significant differences between scan and registration method. Thus, good results can be achieved with CBCT, if it is accessible as intraoperative setup and there is no need to transfer the patient from the operating theater to the radiology department during anesthesia.

The surface registration results were also within an acceptable range. The results showed a higher state of accuracy with FM registration than surface registration, compared to Schicho's study (14), yet there was no statistically significant difference. The current research used the touch surface registration on the lateral side of the head (mastoid to temporal area). In optoelectronic IGS, the laser surface registration method is widely used in ENT surgery (mainly rhinology and anterior skull base) (11), yet this method is not available for electromagnetic IGS systems as performed in this study. The probe tip is fine and could be used easily and safely.

The best accuracy rates were recorded on the facial nerve and at Lat. SCC in the mastoid approach and GG in the MCF approach, and the lowest accuracies were in the lower cranial nerve and trigeminal ganglion area (1.6 to 1.8 $\mathrm{mm}$ error). This means that the accuracy diminishes in distant areas, as mentioned by Ledderose et al. (15).

The researchers used three fiducial markers with acceptable error. However, Grayeli et al. recommended using a combination of skin contouring and fiducial registration for temporal bone surgery (11). The accuracy in the IAC with a retrosigmoid approach was about $1 \mathrm{~mm}$, a little lower than Samii's (16) report.

In this study, the researchers used Cone Beam Computed Tomography (CBCT) or Digital Volume Tomography (DVT), as a portable intraoperative volume scanner to provide reformatted images in the axial, coronal, and sagittal planes within one minute (17). The scanned images could be used in navigation systems in the anterior and lateral skull base $(18,19)$.

The system was user friendly and without any complexity. No interference with the working field was noted during the procedure. This study did not observe any significant distortions by metallic instruments within the surgical field. Setting up the system is very simple and fast; the electromagnetic sensor is used as the navigation head rest beneath the head of the patient. In addition, the electromagnetic head reference frame sensor can be fixed to the head with a centrally placed screw or alternatively with a head band.

This study showed that the electromagnetic navigation system could be used with acceptable accuracy in lateral skull base surgery, especially to localize structures not so deep. Better accuracy could be achieved with FM registration and intra-operative imaging; however, surface registration is also acceptable.

The limitation of the study was the limited number of samples and in some cases, missing some target points that had been previously damaged in education. Clinical application of this system at the lateral skull base surgery in the future could show more details.

\subsection{Conclusion}

Using a navigation system in the lateral skull base is fairly straightforward and feasible. It should be noted, however, that the accuracy decreases through increasing distance from the registration points. However, intratemporal application of this technology is sufficiently accurate and practical.

\section{Acknowledgments}

The second author, DM, was the Chief Technology Officer at Fiagon, yet the other authors declare that they did 


\section{Accuracy in Different Marking Points}

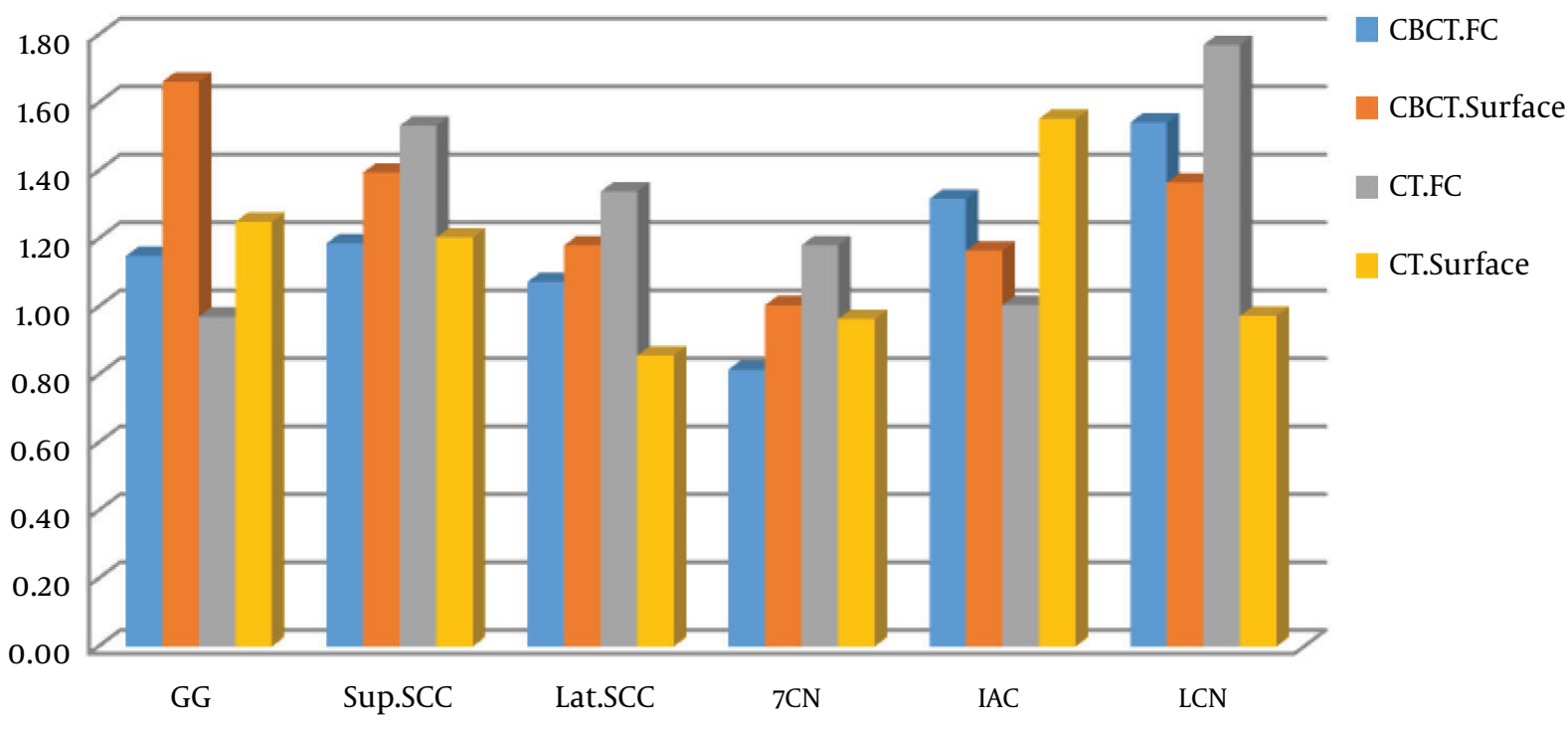

Figure 4. Mean accuracy of different imaging and registration protocols on the different, target points

Mean Accuracy on Different Point of Iterest in All Method

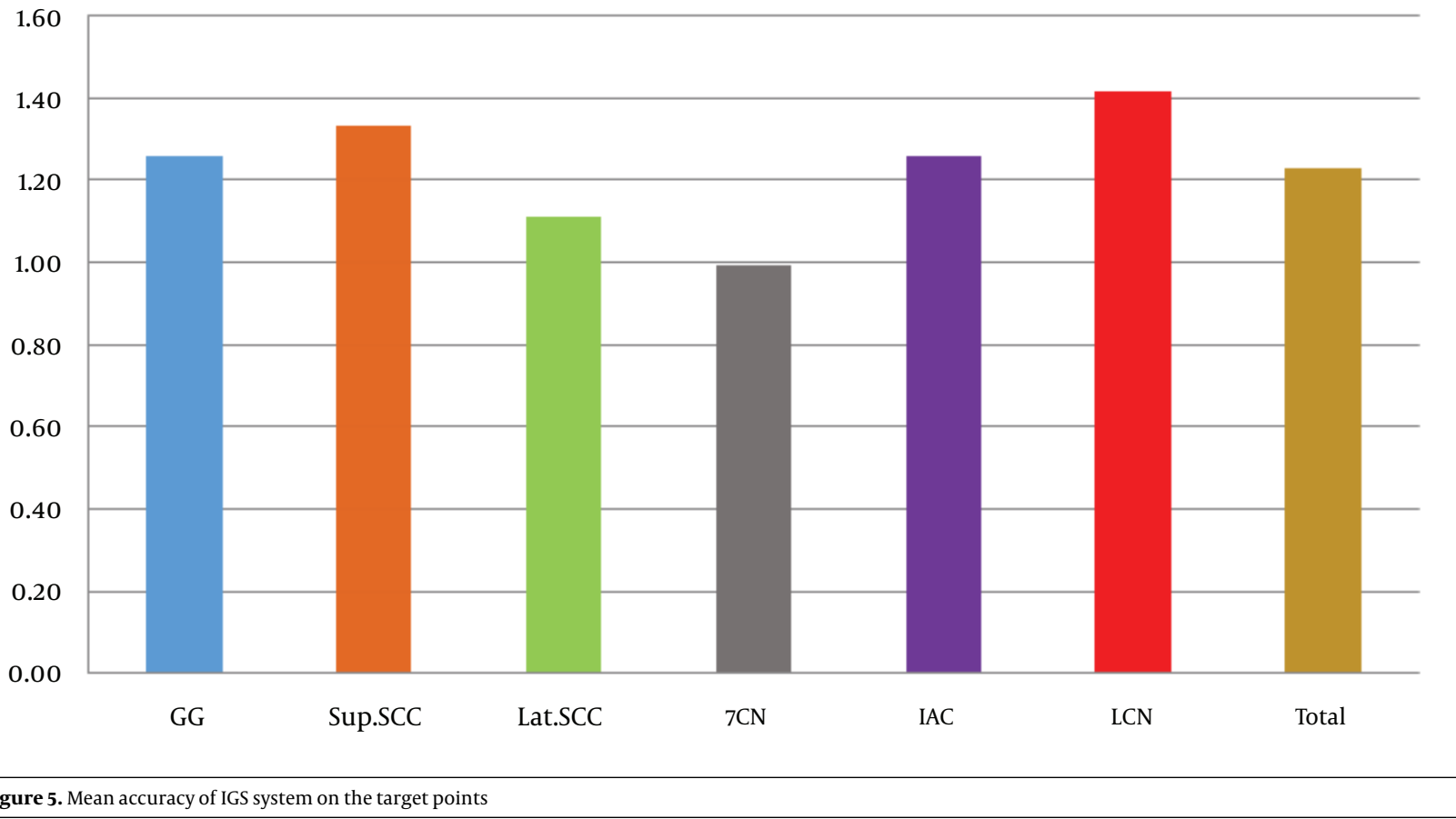

Figure 5. Mean accuracy of IGS system on the target points

not have any relation and conflict of interest with this company. They assisted in hardware preparation, study process, and data analysis. The authors thank Mr. Imanol
Obiri, Fiagon GMBH, Berlin, for support in processing the data. The authors also thank Dr. Donald Giovanetto for his great effort in linguistic editing of the manuscript. 


\section{References}

1. Casiano RR, Numa WA. Efficacy of Computed Tomographic Image???Guided Endoscopic Sinus Surgery in Residency Training Programs. The Laryngoscope. 2000;110(8):1277-82. doi: 10.1097/00005537200008000-00010.

2. Weinberg JS, Lang FF, Sawaya R. Surgical management of brain metastases. Current Oncology Reports. 2001;3(6):476-83. doi: 10.1007/s11912001-0068-6.

3. Metzger MC, Rafii A, Holhweg-Majert B, Pham AM, Strong B. Comparison of 4 Registration Strategies for Computer-Aided Maxillofacial Surgery. Otolaryngology-Head and Neck Surgery. 2016;137(1):93-9. doi: 10.1016/j.otohns.2007.02.015.

4. Kristin J, Mucha D, Schipper J, Klenzner T. Registrierstrategien für die Anwendung des Navigationssystems FIAGON an der lateralen Schädelbasis. Laryngo-Rhino-Otologie. 2012;91(5):306-10. doi:10.1055/s0031-1299755.

5. Grauvogel TD, Soteriou E, Metzger MC, Berlis A, Maier W. Influence of different registration modalities on navigation accuracy in ear, nose, and throat surgery depending on the surgical field. The Laryngoscope. 2010:NA. doi: 10.1002/lary.20867.

6. Kral F, Puschban EJ. Comparison of optical and electromagnetic tracking for navigated lateral skull base surgery. Int J Med Robotic Comput Assist Surg. 2013;9(2):247-52.

7. Schlosser RJ, Bolger WE. Image-Guided Procedures of the Skull Base. Otolaryngologic Clinics of North America. 2005;38(3):483-90. doi: 10.1016/j.otc.2004.10.020

8. Pillai P, Sammet S, Ammirati M. Application Accuracy of Computed Tomography-Based, Image-Guided Navigation of Temporal Bone. Operative Neurosurgery. 2008;63:326-33. doi: 10.1227/01.neu.0000316429.19314.67.

9. Balachandran R, Fitzpatrick J, Labadie RF. Accuracy of Image-Guided Surgical Systems at the Lateral Skull Base as Clinically Assessed Using Bone-Anchored Hearing Aid Posts as Surgical Targets. Otology \& Neurotology. 2008;29(8):1050-5. doi: 10.1097/MAO.0b013e3181859a08.

10. Bernardeschi D, Nguyen Y, Villepelet A, Ferrary E, Mazalaigue $S$, Kalamarides $M$, et al. Use of bone anchoring device in elec- tromagnetic computer-assisted navigation in lateral skull base surgery. Acta Oto-Laryngologica. 2013;133(10):1047-52. doi: 10.3109/00016489.2013.808764

11. Grayeli AB, Esquia-Medina G, Nguyen Y, Mazalaigue S, Vellin J, Lombard B, et al. Use of anatomic or invasive markers in association with skin surface registration in image-guided surgery of the temporal bone. Acta Oto-Laryngologica. 2009;129(4):405-10. doi: $10.1080 / 00016480802579025$.

12. J Muhling RMGE. Laser surface registration for lateral skull base surgery. Bibliogr Minim Invas Neurosurg. 2005;48(3):181-5.

13. Staecker H, O'Malley BW, Eisenberg H, Yoder B. Use of the LandmarX ${ }^{\mathrm{TM}}$ Surgical Navigation System in Lateral Skull Base and Temporal Bone Surgery. Skull Base. 2001;11(4):245-56. doi: 10.1055/s-2001-18631.

14. Schicho K, Figl M, Seemann R, Donat M, Pretterklieber ML, Birkfellner $W$, et al. Comparison of laser surface scanning and fiducial marker-based registration in frameless stereotaxy. Journal of Neurosurgery. 2007;106(4):704-9. doi:10.3171/jns.2007.106.4.704.

15. Ledderose GJ, Stelter K, Leunig A, Hagedorn H. Surface laser registration in ENT-surgery: accuracy in the paranasal sinuses-a cadaveric study. Rhinology. 2007;45(4):281-5. [PubMed: 18085021].

16. Samii A, Brinker T, Kaminsky J, Lanksch WR, Samii M. Navigationguided Opening of the Internal Auditory Canal via the Retrosig moid Route for Acoustic Neuroma Surgery: Cadaveric, Radiological and Preliminary Clinical Study. Neurosurgery. 2000;47(2):382-8. doi: 10.1097/00006123-200008000-00021.

17. De Vos W, Casselman J, Swennen GRJ. Cone-beam computerized tomography (CBCT) imaging of the oral and maxillofacial region: A systematic review of the literature. International Journal of Oral and Maxillofacial Surgery. 2009;38(6):609-25. doi:10.1016/j.ijom.2009.02.028.

18. Labadie RF, Majdani O, Fitzpatrick J. Image-Guided Technique in Neurotology. Otolaryngologic Clinics of North America. 2007;40(3):611-24 doi:10.1016/j.otc.2007.03.006.

19. Bartling SH, Leinung $\mathrm{M}$, Graute J, Rodt $\mathrm{T}$, Dullin $\mathrm{C}$, Becker $\mathrm{H}$, et al. Increase of Accuracy in Intraoperative Navigation Through High-Resolution Flat-Panel Volume Computed Tomography. Otology \& Neurotology. 2007;28(1):129-34. doi: 10.1097/01.mao.0000244364.16826.09. 